Pacific Journal of Mathematic 


\title{
ON THE STRUCTURE OF INFINITELY DIVISIBLE DISTRIBUTIONS
}

\author{
J. R. Blum and M. Rosenblatt
}

1. Introduction and summary. Let $F(x)$ be a distribution on the real line. Then we may write

$$
F(x)=p F_{1}(x)+(1-p) F_{2}(x)
$$

where $F_{1}(x)$ is a discrete distribution, $F_{2}(x)$ is a continuous distribution and $0 \leq p \leq 1$. We shall say that $F(x)$ is discrete if $p=1, F(x)$ is continuous if $p=0$ and $F(x)$ is a mixture if $0<p<1$.

Let $\varphi(s)=\int_{-\infty}^{\infty} e^{i s x} d F(x)$ be the characteristic function corresponding to $F(x)$. It would be useful to give a convenient criterion on $\varphi(s)$ to determine when the corresponding distribution $F(x)$ is discrete, continuous, or a mixture. In $\S 2$ we give such a criterion for the class of infinitely divisible (i.d.) distributions, utilizing the Khinchin representation of the characteristic function of such a distribution. In $\S 3$ we apply the theorem of $\S 2$ to characterize a certain class of stochastic processes.

2. The structure theorem. Let $\varphi(s)$ be the characteristic function of an i.d. distribution. The Khinchin representation of such a characteristic function takes the form

$$
\varphi(s)=\exp \left\{i \gamma s+\int_{-\infty}^{\infty}\left[e^{i u s}-1-\frac{i u s}{1+u^{2}}\right] \frac{1+u^{2}}{u^{2}} d G(u)\right\}
$$

where $\gamma$ is a real number and $G(u)$ is a real valued bounded nondecreasing function. $\gamma$ and $G(u)$ are uniquely determined by the conditions $G(-\infty)=0, G(u+0)=G(u)$. We shall need the following two lemmas, the first of which is well known.

Lemma 1. Let $X$ and $Y$ be independent random variables. Then

(i) the distribution of $X+Y$ is discrete if and only if the distribution of each of the variables is discrete,

(ii) the distribution of $X+Y$ is a mixture if and only if one of the two distributions is a mixture and the other is either discrete or a mixture.

Let $F(x)$ be a distribution. We shall define $F^{(k)}(x)$ as follows :

Received July 16, 1958. This research was sponsored by the Office of Ordnance Research, U. S. Army and the Office of Naval Research, U. S. Navy. Reproduction in whole or in part is permitted for any purpose of the U. S. Government. 


$$
F^{(0)}(x)=\left\{\begin{array}{ll}
0 & \text { for } x<0 \\
1 & \text { for } x \geq 0
\end{array}, F^{(1)}(x)=F(x),\right.
$$

and for $k \geq 2, F^{(k)}(x)$ denotes the $k$-fold convolution of $F(x)$ with itself.

Lemma 2. Let $-\infty \leq a<b \leq \infty$, and let $F(x)$ be a nondecreasing, bounded function defined for $a \leq x \leq b$ such that $F(a)=0, F(b)-F(a)=$ $c>0$. Then

$$
\varphi(s)=\exp \left\{-c+\int_{a}^{b} e^{i s x} d F(x)\right\}
$$

is a characteristic function corresponding to the i.d. distribution

$$
H(u)=e^{-c} \sum_{k=0}^{\infty} \frac{F^{(k)}(u)}{k !} .
$$

If $F(x)$ is a pure jump function then $H(u)$ is discrete. If $F(x)$ is continuous, then $H(u)$ is a mixture with a jump of magnitude $e^{-c}$ at the origin and continuous otherwise.

Proof. For every positive integer $n$ let

$$
H_{n}(u)=\sum_{k=0}^{n} \frac{F^{(k)}(u)}{k !} / \sum_{k=0}^{n} \frac{c^{k}}{k !}
$$

and let

$$
\varphi_{n}(s)=\sum_{k=0}^{n} \frac{1}{k !}\left[\int_{a}^{b} e^{i s k} d F(x)\right]^{k} / \sum_{k=0}^{n} \frac{c^{k}}{k !} .
$$

Then $H_{n}(u)$ is a distribution with characteristic function $\varphi_{n}(s)$. Since $H_{n}(u)$ converges to $H(u)$ and $\varphi(s)$ converges to the continuous function $\varphi(s)$ it follows that $H(u)$ is a distribution with characteristic function $\varphi(s)$. The fact that $H(u)$ is i.d. is immediate from the form of $\varphi(s)$. Now if $F(x)$ is a pure jump function then (2.2) becomes

$$
\varphi(s)=e^{-c} \sum_{k=0}^{\infty} \frac{1}{k !}\left[\sum_{j=1}^{\infty} e^{i s x_{j}} p_{j}\right]^{k}
$$

where $F(x)$ has its jumps at the points $x_{j}$ with magnitudes $p_{j}$, and such a characteristic function clearly corresponds to a discrete distribution. Finally if $F(x)$ is continuous we may write

$$
H(u)=e^{-c} F^{(0)}(u)+\frac{\left(1-e^{-c}\right)}{\left(e^{c}-1\right)} \sum_{k=1}^{\infty} \frac{F^{(k)}(u)}{k !},
$$

and since the infinite series converges uniformly it follows that $H(u)$ is the mixture of a continuous distribution and the distribution with a single jump at zero. 
Theorem 1. Let $\varphi(s)$ be the characteristic function of an i.d. distribution $F(x)$. Let $G(u)$ bo the function occurring in the representation (2.1). Then

(i) $F(x)$ is discrete if and only if $\int_{-\infty}^{\infty} \frac{1}{u^{2}} d G(u)<\infty$ and $G(u)$ is a pure jump function.

(ii) $F(x)$ is a mixture if and only if $\int_{-\infty}^{\infty} \frac{1}{u^{2}} d G(u)<\infty$ and $G(u)$ is not a pure jump function

(iii) $F(x)$ is continuous if and only if $\int_{-\infty}^{\infty} \frac{1}{u^{2}} d G(u)=\infty$.

Proof. Suppose first that $G(u)$ is a pure jump function with jumps at the points $u_{j}, j=1,2, \cdots$ and with corresponding magnitudes $\rho_{j} \geq 0$, such that $\sum_{j} \rho_{j}<\infty$. Then (2.1) (with $\gamma=0$ ) takes the form

$$
\varphi(s)=\exp \left\{\sum_{j}\left[e^{i s u_{j}}-1-\frac{i s u_{j}}{1+u_{j}^{2}}\right] \frac{1+u_{j}^{2}}{u_{j}^{2}} \rho_{j}\right\} .
$$

Now if $\sum_{j} \rho_{j} / u_{j}^{2}<\infty$ we may rewrite (2.4) in the form

$$
\varphi(s)=\exp \left\{i s b-c+\int_{-\infty}^{\infty} e^{i s u} d M(u)\right\}
$$

where

$$
b=-\sum_{j} \frac{\rho_{j}}{u_{j}}, c=\sum_{j} \frac{1+u_{j}^{2}}{u_{j}^{2}} \rho_{j},
$$

and where $M(u)$ is a bounded, nondecreasing, pure jump function with jumps at the points $u_{j}$ and corresponding magnitudes $\left(\left(1+u_{j}^{2}\right) / u_{j}^{2}\right) \rho_{j}$. Consequently it follows from Lemmas 1 and 2 that $F(x)$ is discrete.

Conversely we suppose that $F(x)$ is a discrete distribution. We shall show first that $G(u)$ is a pure jump function. To do this write $G(u)=$ $G_{1}(u)+G_{2}(u)$ where $G_{1}(u)$ is a pure jump function and $G_{2}(u)$ is continuous. If $G(u)$ is not a pure jump function there will exist a closed interval $[a, b]$ not containing zero such that $G_{2}(a)<G_{2}(b)$. Then we may write $\varphi(s)$ in the form $\varphi(s)=M(s) N(s)$ where $M(s)$ is a characteristic function and

$$
\begin{aligned}
N(s) & =\exp \left\{\int_{a}^{b}\left[e^{i s u}-1-\frac{i s u}{1+u^{2}}\right] \frac{1+u^{2}}{u^{2}} d G_{2}(u)\right\} \\
& =\exp \left\{-i s \int_{a}^{b} \frac{1}{u} d G_{2}(u)-\int_{a}^{b} \frac{1+u^{2}}{u^{2}} d G_{2}(u)+\int_{a}^{b} e^{i s u} d H(u)\right\}
\end{aligned}
$$

where $d H(u)=\left(\left(1+u^{2}\right) / u^{2}\right) d G_{2}(u)$. From Lemma 2 it follows that $N(s)$ is the characteristic function of a mixture and from Lemma 1 it then 
follows that $F(x)$ is not discrete. Hence $G(u)$ is a pure jump function, and $\varphi(s)$ has the form (2.4).

We shall show that $\sum_{j} \rho_{j} / u_{j}^{2}<\infty$. Since $\sum_{j} \rho_{j}<\infty$ it is sufficient to restrict attention to those $u_{j}$ for which $\left|u_{j}\right| \leq 1$. Since $F(x)$ is discrete it follows that $\varphi(s)$ is almost periodic and we have

$$
\lim _{R \rightarrow \infty} \frac{1}{R} \int_{0}^{R}|\varphi(s)|^{2} d s>0
$$

Now

$$
|\varphi(s)|^{2}=\exp \left\{\sum_{j}\left[\cos u_{j} s-\lambda 1\right] \frac{\lambda_{j}}{u_{j}^{2}}\right\}
$$

where

$$
\lambda_{j}=2\left[1+u_{j}^{2}\right] \rho_{j} .
$$

Let

$$
g(R)=\sum_{1 / R \leq\left|u_{j}\right| \leq 1} \frac{\lambda_{j}}{u_{j}^{2}}
$$

We have

$$
\begin{aligned}
|\varphi(s)|^{2} & \leq \exp \left\{\sum_{1 / R \leq\left|u_{j}\right| \leq 1}\left[\cos u_{j} s-\right] \frac{\lambda_{j}}{u_{j}^{2}} g(R) / g(R)\right\} \\
& \leq \frac{1}{g(R)} \sum_{1 / R \leq\left|u_{j}\right| \leq 1} \frac{\lambda_{j}}{u_{j}^{2}} \exp \left\{\left[\cos u_{j} s-1\right] g(R)\right\} .
\end{aligned}
$$

The first of these inequalities is immediate and the second is an application of Jensen's inequality.

From (2.6) we obtain

$$
\begin{gathered}
\frac{1}{R} \int_{0}^{R}|\varphi(s)|^{2} d s \\
\leq \frac{1}{g(R)} \sum_{1 / R \leq\left|u_{j}\right| \leq 1} \frac{\lambda_{j}}{u_{j}^{2}} \frac{1}{R} \int_{0}^{R} \exp \left\{\left[\cos u_{j} s-1\right] g(R)\right\} d s .
\end{gathered}
$$

Suppose $R \geq 1$ and $\left|u_{j}\right| \geq 1 / R$. Then for every $\varepsilon>0$ there exists $\delta$ depending on $\varepsilon$ only with $0<\delta<1$ and with the following property: If $R_{1}(\varepsilon)$ is the subset of $[0, R]$ where $\cos u_{j} s<1-\delta$ and $R_{2}(\varepsilon)$ is the subset of $[0, R]$ where $\cos u_{j} s \geq 1-\delta$, then the measure of $R_{2}(\varepsilon)$ does not exceed $\varepsilon R$. Using this and (2.7) we find

$$
\frac{1}{R} \int_{0}^{R}|\varphi(s)|^{2} d s \leq \varepsilon+e^{-\delta g(R)} .
$$


Now if $\sum_{j} \rho_{j} / u_{j}^{2}=\infty$, then clearly $\lim _{R \rightarrow \infty} g(R)=\infty$. This together with (2.8) contradicts (2.5), thus proving (i).

Now suppose $\int_{-\infty}^{\infty} 1 / u^{2} d G(u)<\infty$ and $G(u)$ is not a pure jump function. Then we may write $G(u)=G_{1}(u)+G_{2}(u)$ where $G_{1}(u)$ is a pure jump function and $G_{2}(u)$ is continuous. Of course we have

$$
\int_{-\infty}^{\infty} \frac{1}{u^{2}} d G_{i}(u)<\infty, \quad i=1,2 .
$$

Then from (i)

$$
\exp \left\{\int_{-\infty}^{\infty}\left[e^{i s u}-1-\frac{i s u}{1+u^{2}}\right] \frac{1+u^{2}}{u^{2}} d G_{1}(u)\right\}
$$

is the characteristic function of a discrete distribution. Similarly from Lemma 2 it follows that

$$
\exp \left\{\int_{-\infty}^{\infty}\left[e^{i s u}-1-\frac{i s u}{1+u^{2}}\right] \frac{1+u^{2}}{u^{2}} d G_{2}(u)\right\}
$$

is the characteristic function of a mixture. Thus $F(x)$ is the convolution of a discrete distribution and a mixture and from Lemma 1 it follows that $F(x)$ is a mixture.

Conversely suppose $F(x)$ is a mixture. Then

$$
\varphi(s)=p \varphi_{1}(s)+(1-p) \varphi_{2}(s)
$$

where $0<p<1, \varphi_{1}(s)$ is the characteristic function of a discrete distribution and $\varphi_{2}(s)$ is the characteristic function of a continuous distribution. If we write $\varphi(s)=e^{\psi(s)}$ then $e^{\psi(s) / n}$ is a characteristic function for every positive integer $n$ because $F(x)$ is infinitely divisible. Clearly $e^{\psi(s) / n}$ must be the characteristic function of a mixture, i.e.

$$
e^{\psi(s) / n}=p_{n} \varphi_{1, n}(s)+\left(1-p_{n}\right) \varphi_{2, n}(r)
$$

where $0<p_{n}<1$, and $\varphi_{1, n}(s)$ and $\varphi_{2, n}(s)$ are of the same type as $\varphi_{1}(s)$ and $\varphi_{2}(s)$ respectively. From (2.9) and (2.10) we obtain

$$
\begin{aligned}
\varphi(s) & =\left[e^{\frac{\psi(s)}{n}}\right]^{n}=p_{n}^{n} \varphi_{1, n}^{n}(s) \\
& +\sum_{k=1}^{n}\left(\begin{array}{l}
n \\
k
\end{array}\right) p_{n}^{n-k}\left(1-p_{n}\right)^{k} \varphi_{1, n}^{n-k}(s) \varphi_{2, n}^{k}(s) .
\end{aligned}
$$

Now $\varphi_{1, n}^{n}(s)$ is the characteristic function of a discrete distribution and the sum occurring in (2.11) is the product of $\left(1-p_{n}^{n}\right)$ and a characteristic function of a continuous distribution. Thus $p_{n}=p^{1 / n}$ and $\left[\varphi_{1, n}(s)\right]^{n}=\varphi_{1}(s)$ and we see that $\varphi_{1}(s)$ is the characteristic 
function of an i.d. distribution. Writing $\varphi_{1}(s)=e^{\psi_{1}(s)}, p=e^{-c}$ with $0<c<\infty$ we have

$$
e^{\frac{\psi(s)}{n}}=e^{-\frac{c}{n}} e^{\frac{\varphi_{1}(s)}{n}}+\left(1-e^{-\frac{c}{n}}\right) \varphi_{2, n}(s) .
$$

If we expand the exponentials in (2.12) we obtain

$$
\lim _{n \rightarrow \infty} \varphi_{2, n}(s)=\varphi_{2,0}(s)=1+\frac{\psi(s)-\psi_{1}(s)}{c} .
$$

Since $\psi(s)$ and $\psi_{1}(s)$ are continuous it follows that $\varphi_{2,0}(s)$ is a characteristic function, say $\varphi_{2,0}(s)=\int_{-\infty}^{\infty} e^{i s x} d H(x)$, where $H(x)$ is a distribution. Hence

$$
\begin{aligned}
\varphi(s) & =e^{\psi(s)}=e^{\psi_{1}(s)+\psi(s)-\psi_{1}(s)} \\
& =e^{\psi_{1}(s)+c\left[\varphi_{2,0}(s)-1\right]}=e^{\psi_{1}(s)+} \int_{\infty}^{\infty}\left[e^{i s u}-1\right] a c H(u) .
\end{aligned}
$$

Now $e^{\psi_{1}(s)}$ is the characteristic function of a discrete distribution. If we equate formula (2.14) for $\varphi(s)$ with formula (2.1) for $\varphi(s)$ it follows from the first part of the theorem and the uniqueness of $G(u)$ that $\int_{-\infty}^{\infty} 1 / u^{2} d G(u)<\infty$. It is also a consequence of the first part of the theorem that $G(u)$ is not a pure jump function. Thus (ii) is proved and (iii) follows from (i) and (ii), proving the theorem.

From (2.14) we are able to deduce additional information in the mixed case.

COROLlaRY. Let $\varphi(s)$ be a characteristic function corresponding the i.d. distribution $F(x)$. If $F(x)$ is a mixture then $F(x)$ is the convolution of a discrete i.d. distribution and a i.d. distribution which has a jump at zero of magnitude less than one and is continuous otherwise.

3. A class of discrete processes. Let $X_{j}(t), t \geq 0, j=1,2 \cdots$ be a sequence of independent stochastic processes such that for each $j, X_{j}(t)$ is a process with independent increments and such that for $0 \leq t_{1} \leq t_{2}$ the random variable $X_{j}\left(t_{2}\right)-X_{j}\left(t_{1}\right)$ has characteristic function

$$
\varphi_{j}\left(s, t_{1}, t_{2}\right)=\exp \left\{\left[e^{i s u_{j}}-1-\frac{i s u_{j}}{1+u_{j}^{2}}\right] \frac{1+u_{j}^{2}}{u_{j}^{2}}\left[\rho_{j}\left(t_{2}\right)-\rho_{j}\left(t_{1}\right)\right]\right\}
$$

where $u_{j}$ is a real number and $\rho_{j}(t)$ is a nondecreasing function defined for $t \geq 0$ with $\rho_{j}(0)=0$. Then each $X_{j}(t)$ is a generalized Poisson process, i.e. $X_{j}(t)$ assumes values of the form $y_{k}=k u_{j}-\left(\rho_{j}(t)\right) / u_{j}$ with probability

$$
P\left\{X_{j}(t)=y_{k}\right\}=\frac{e^{-\lambda_{j}(t)} \lambda_{j}^{k}(t)}{k !},
$$


where $\lambda_{j}(t)=\left(\left(1+u_{j}^{2}\right) / u_{j}^{2}\right) \rho_{j}(t)$. Now if $\sum_{j} \rho_{j}(t)<\infty$ for every $t \geq 0$, then we can define a process $X(t)$ as the sum of the processes $X_{j}(t)$, and the characteristic function of the process $X(t)$ will have the form

$$
\varphi(s, t)=\exp \left\{\sum_{j}\left[e^{i s u_{j}}-1-\frac{i s u_{j}}{1+u_{j}^{2}}\right] \frac{1+u_{j}^{2}}{u_{j}^{2}} \rho_{j}(t)\right\} .
$$

It is an immediate consequence of Theorem 1 that for any $t \geq 0, X(t)$ will be a discrete random variable if and only if $\sum_{j}\left(\rho_{j}(t)\right) / u_{j}^{2}<\infty$.

Conversely suppose for $t \geq 0, X(t)$ is a stochastic process such that $X(0)=0, X(t)$ is a discrete random variable for every $t \geq 0$, and the process has independent infinitely divisible increments. This will be true, e.g. if $X(t)$ is a discrete process with independent increments and such that $X(t)$ is continuous in probability. Then from Theorem 1 it follows that the characteristic function of the random variable $X(t)$ is essentially of the form (3.1) with $\rho_{j}(t)$ nondecreasing and $\sum_{j}\left(\rho_{j}(t)\right) / u_{j}^{2}<\infty$ for all $t$. Consequently $X(t)$ has the stochastic structure of a sum of independent generalized Poisson processes. We have

Theorem 2. Let $X(t)$ be a discrete stochastic process for $t \geq 0$, with $X(0)=0$ and such that $X(t)$ has independent infinitely divisible increments. Then there exists a sequence of independent generalized Poisson processes $X_{j}(t), j=1,2 \cdots$ such that $X(t)$ has the same stochastic structure as $\sum_{j} X_{j}(t)$.

In the case when $X(t)$ assumes only integer values Theorem 2 was already proved by Khinchin [1].

\section{REFERENCE}

1. A Khinchin, Nachwirkungsfreie Folgen von zufälligen Ereignissen, Teor. Veroyatnost. i 1, (1956).

INDIANA UNIVERSITY 



\section{PACIFIC JOURNAL OF MATHEMATICS}

\section{EDITORS}

\section{David Gilbarg}

Stanford University

Stanford, California

R. A. Beaumont

University of Washington

Seattle 5 , Washington
A. L. Whiteman

University of Southern California

Los Angeles 7, California

L. J. PAIGE

University of California

Los Angeles 24, California

\section{ASSOCIATE EDITORS}
E. F. BECKENBACH
C. E. BURGESS
E. HEWITT
A. HORN

\author{
V. GANAPATHY IYER \\ R. D. JAMES \\ M. S. KNEBELMAN \\ L. NACHBIN
}
I. NIVEN
E. G. STRAUS
T. G. OSTROM
H. L. ROYDEN
G. SZEKERES
M. M. SCHIFFER
F. WOLF
K. YOSIDA

\section{SUPPORTING INSTITUTIONS}

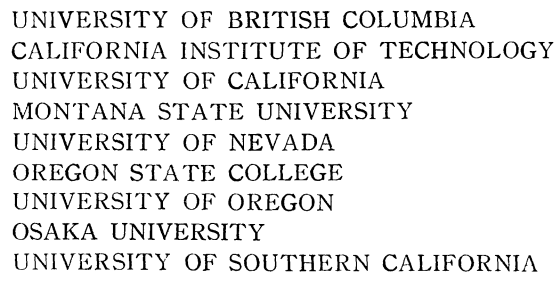

UNIVERSITY OF BRITISH COLUMBIA CALIFORNIA INSTITUTE OF TECHNOLOGY UNIVERSITY OF CALIFORNIA MONTANA STATE UNIVERSITY UNIVERSITY OF NEVADA OREGON STATE COLLEGE UNIVERSITY OF OREGON OSAKA UNIVERSITY UNIVERSITY OF SOUTHERN CALIFORNIA

STANFORD UNIVERSITY

UNIVERSITY OF TOKYO

UNIVERSITY OF UTAH

WASHINGTON STATE COLLEGE

UNIVERSITY OF WASHINGTON

AMERICAN MATHEMATICAL SOCIETY CALIFORNIA RESEARCH CORPORATION HUGHES AIRCRAFT COMPANY SPACE TECHNOLOGY LABORATORIES

Printed in Japan by Kokusai Bunken Insatsusha (International Academic Printing Co., Ltd.), Tokyo, Japan 


\section{Pacific Journal of Mathematics}

\section{Vol. 9, No. 1 \\ May, 1959}

Julius Rubin Blum and Murray Rosenblatt, On the structure of infinitely

divisible distributions . ............................. 1

Robert Geroge Buschman, Asymptotic expressions for

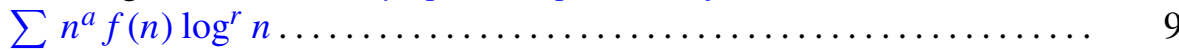

Eckford Cohen, A class of residue systems $(\bmod r)$ and related arithmetical

functions. I. A generalization of Möbius inversion .............. 13

Paul F. Conrad, Non-abelian ordered groups ................... 25

Richard Henry Crowell, On the van Kampen theorem............... 43

Irving Leonard Glicksberg, Convolution semigroups of measures ........ 51

Seymour Goldberg, Linear operators and their conjugates ............ 69

Olof Hanner, Mean play of sums of positional games .............. 81

Erhard Heinz, On one-to-one harmonic mappings ................ 101

John Rolfe Isbell, On finite-dimensional uniform spaces . . ........... 107

Erwin Kreyszig and John Todd, On the radius of univalence of the function

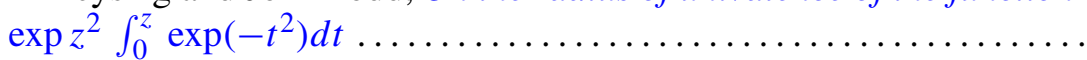

Roger Conant Lyndon, An interpolation theorem in the predicate

calculus......................................... 129

Roger Conant Lyndon, Properties preserved under homomorphism ........ 143

Roger Conant Lyndon, Properties preserved in subdirect products ....... 155

Robert Osserman, A lemma on analytic curves ................ 165

R. S. Phillips, On a theorem due to Sz.-Nagy..................... 169

Richard Scott Pierce, A generalization of atomic Boolean algebras ....... 175

J. B. Roberts, Analytic continuation of meromorphic functions in valued fields................................. 183

Walter Rudin, Idempotent measures on Abelian groups ................ 195

M. Schiffer, Fredholm eigen values of multiply-connected domains ........ 211

V. N. Singh, A note on the computation of Alder's polynomials ......... 271

Maurice Sion, On integration of 1-forms ...................... 277

Elbert A. Walker, Subdirect sums and infinite Abelian groups........... 287

John W. Woll, Homogeneous stochastic processes . .................. 293 